\title{
Instrument Requirements for Eddy Correlation Measurements
}

\author{
Gordon A. McBean \\ Atmospheric Jinvironment Service, Toronto, Ontario, Canada
}

(Manuscript received 28 February 1972, in revised form 13 July 1972)

\section{ABSTRACT}

\begin{abstract}
The response requirements of the sensors, in particular the vertical wind sensor, used for eddy correlation measurements are discussed. Data are presented to allow estimation of the errors due to inadequate cosine and frequency response of the vertical wind sensor. A Gill propeller anemometer is examined as an example to show how large the errors might be.
\end{abstract}

\section{Introduction}

The turbulent eddy fluxes play an important role in the dynamics and thermodynamics of the atmospheric surface layer. The problems of directly determining these fluxes have been investigated by several research groups over the last two decades and considerable success has been achieved. In addition to these specialized research groups, scientists in agriculture, forestry, urban studies, and other fields are interested in the results of these types of measurements. As part of the Global Atmospheric Research Program, there is a need for flux measurements over a wide variety of earth surfaces. The object of this paper is to discuss some important aspects of eddy correlation measurements that must be considered in choosing experimental equipment and in interpreting the results.

It will be assumed that the measurements are to be made at one point as a function of time. A most important aspect to consider is the ability of the sensor to respond to the range of frequencies that contribute to the flux. This will be discussed first. For vertical flux measurements the critical sensor is the vertical wind anemometer which must be able to correctly distinguish between the vertical component and the total wind vector. Thus, the wind angle-of-attack response will be discussed secondly. In the last section, the Gill propeller anemometer will be discussed as an example of a vertical wind sensor. It will be shown how to determine the likely error in the flux.

This discussion will not explicitly cover the other sensor, be it a thermometer, humidiometer, or whatever, that is also required for flux measurement. With the exception of momentum flux studies, the other sensor will be measuring a scalar quantity and the main criteria are that it have sufficient resolution and frequency response. The discussion on frequency response that follows is equally pertinent to the other sensor as to the vertical wind sensor.

\section{Frequency response}

In many considerations of the governing equations for turbulent flow terms of the form $\overline{w \alpha}$ are important in the equations for the mean parameters [see Lumley and Panofsky (1964) for further information]. Here $w$ is the vertical component of the turbulent wind and $\alpha$ is the deviation from the mean of some variable such as horizontal wind, temperature, humidity, etc. The overbar signifies an average over time, which will be assumed to be equal to the ensemble average.

The average $\overrightarrow{w \alpha}$ can be considered as the superposition of oscillations of $w$ and $\alpha$ of all frequencies such that

$$
\overline{w \alpha}=\frac{1}{T} \int_{0}^{T} w \alpha d t=\int_{0}^{\infty} \phi_{w \alpha}(n) d n,
$$

where $n$ is frequency $(\mathrm{Hz}), T$ the length of the time average, and $\phi_{w \alpha}(n)$ the cospectrum of $w$ and $\alpha$. The difficulty with this integration over frequency is that atmospheric oscillations have contributions from all frequencies including diurnal or longer period oscillations. It is usual to impose a lower limit $n_{L}$ on the integration over frequency. Because of the nature of turbulence, the function $\phi_{w \alpha}(n)$ will also usually become near zero for frequencies beyond an upper limit $n_{l}$. Hence we can redefine the average as

$$
\overline{w \alpha}=\int_{n_{L}}^{n_{h}} \phi_{w \alpha}(n) d n=\int_{\ln n_{L}}^{\ln n_{\Lambda}} n \phi_{w \alpha}(n) d \ln n,
$$

and both $n_{h}$ and $n_{L}$ should be determined by examining $\phi_{w \alpha}(n)$. The average represented by (2) will usually be somewhat different from that of (1). Use of (2) to compute the fluxes requires that the turbulence signals of $v$ and $\alpha$ be recorded and the cospectrum produced, examined and then integrated. This is a time-consuming and expensive operation and is probably not justified for many applications. From the practical point of view 
it is better to compute the time average of the product of $w$ and $\alpha$ in real time either by analog or digital means (see, e.g., Hicks, 1970). However, unless the turbulence signals are filtered correctly a true representation of (2) will not be achieved.

For flow over homogeneous terrain with steady-state conditions, similarity theory (Monin and Obukhov, 1954; Lumley and Panofsky, 1964) predicts that these cospectra should have a standard form which is a function of $z / L$, the Monin-Obukhov stability parameter. It is predicted that

$$
n \phi_{w \alpha}(n)=\bar{w} \alpha \psi_{w \alpha}(f, z / L),
$$

where $f=n z / u$ is the nondimensional frequency, $\psi(f, z / L)$ the standard form, $z$ the height of observation, $u$ the mean wind speed at height $z$, and $L$ the MoninObukhov length $u_{*}^{3} T /\left(k g \overline{v^{\prime} T^{\prime}}\right)$. Recent results by McBean and Miyake (1972), which are for measurements $2 \mathrm{~m}$ above a grassy surface, are shown in Fig. 1 . These cospectra agree with those of Miyake et al. (1970) from over water and Pond et al. (1971) from BOMEX (except for heat flux). Kaimal et al. (1972) report that the cospectral results from their Kansas experiment agree well with these cospectra. The agreement with an empirical form proposed by Panofsky and Mares (1968) and based on earlier results is not as good, as is indicated in Fig. 1.

Using Fig. 1, the upper and lower limits of integration can be determined if the wind speed, height of observation, and stability are known. By considering the range of wind speeds and stabilities to be encountered, it is possible to pick the best height for the measurements in order that the sensors can cover most of the range of frequencies required. Note that these results are for homogeneous terrain and steady-state conditions, and may not be applicable to other conditions.

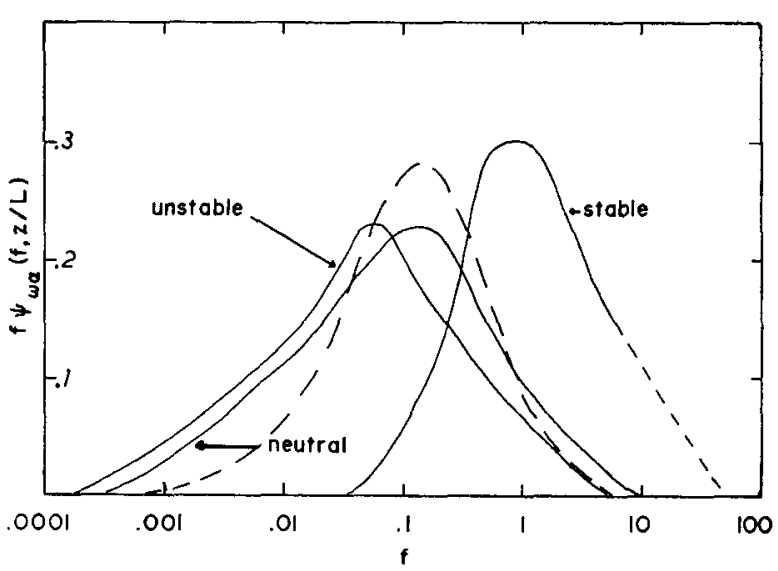

FIG. 1. The normalized logarithmic cospectra for $w$ and $\alpha$, where $\alpha$ may be the horizontal wind, temperature or specific humidity, for stable $(4<z / L<10)$, neutral $(-0.04<z / L<0.1)$ and unstable $(-0.46>z / L>-0.63)$ stratifications (after McBean and Miyake, 1972). The dashed curve is the form proposed by Panofsky and Mares (1968).

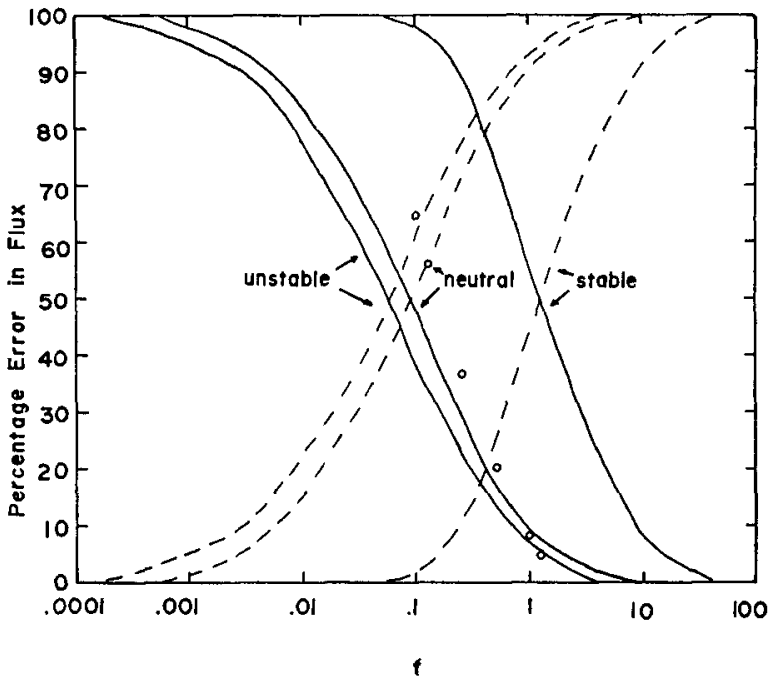

Fig. 2. The percentage of the flux lost, or percentage error, due to limitations in frequency response. The solid curves give the error due to high-frequency cut-off while the dashed curves give the error for low-frequency cut-off. Stable $(z / L \approx 5)$, neutral, and unstable $(z / L \approx-0.5)$ stratifications are presented. Some results from Deacon (1959) for the high-frequency cut-off error for neutra? conditions are indicated by the open circles.

Because of limitations on frequency response of the sensors or recording equipment or limitations on the length of observations, some part of the range of frequencies of interest will necessarily be missed. The cospectra presented in Fig. 1 can be used to compute the error in the estimated fluxes. For example, if the system has a high frequency cut-off at $f_{h}{ }^{\prime}$ (which corresponds to a $n_{h}{ }^{\prime}$ for given wind and height conditions) then the ratio of the measured flux to the true flux will be the ratio of the integral under the appropriate cospectrum of Fig. 1 from $j_{L}$ to $f_{h}^{\prime}$ compared to the integral from $f_{L}$ to $f_{h}$. The percentage errors due to both high- and low-frequency cut-offs are given in Fig. 2. Note that an idealized filter with unit response above (below) and zero response below (above) the cut-off frequency was assumed. For an RC type filter the errors would be slightly larger. Because the variation with stability (for unstable conditions) of the cospectrum is small, one correction factor as a function of wind speed alone would probably suffice for most applications. For stable cases more care in correcting the fluxes is required. Also given on Fig. 2 are some results of Deacon (1959) for the neutral case. His results are somewhat different.

\section{Angle-of-attack response}

In addition to being able to respond to a wide range of frequencies, the vertical wind sensor must be able to respond correctly to a wide range of angles of attack. The angle of attack is the angle between the instantaneous total wind vector and the horizontal plane. In Fig. 3, the fractional contributions to the total momentum or heat transfer for different angles of attack 


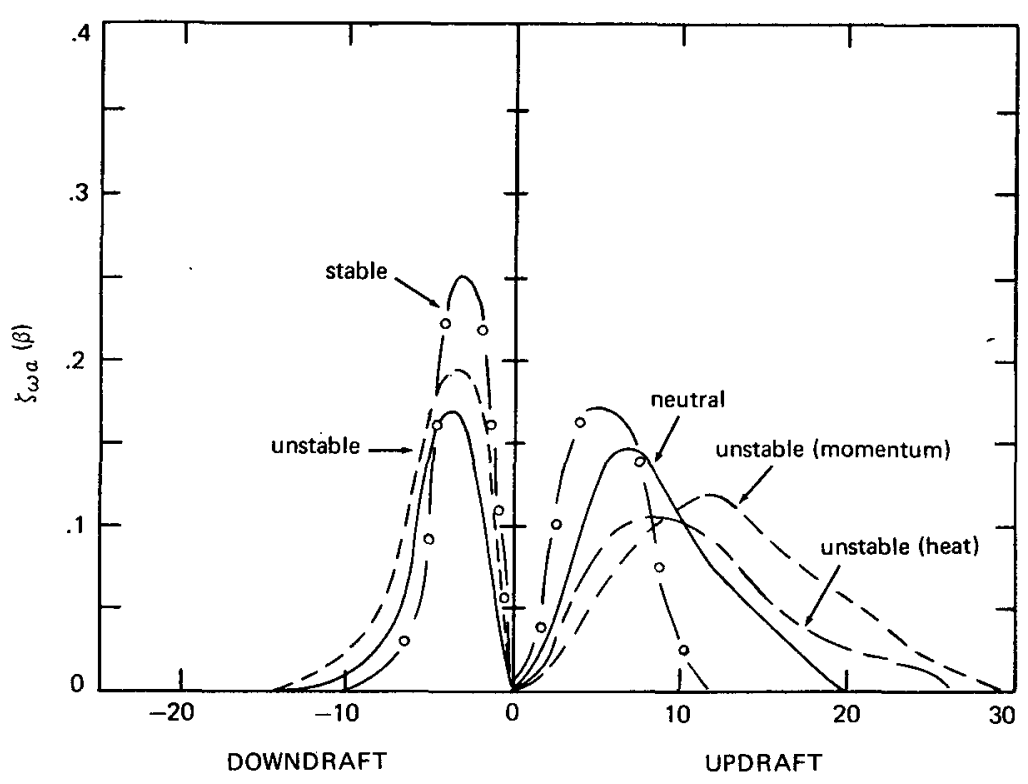

$\beta$ (degrees)

FIG. 3. The flux-angle distributions for stable $(z / L \approx 5)$, near neutral, and unstable $(z / L \approx-0.5)$ stratifications. The flux-angle distributions give the fraction of the total flux transferred when the angle of attack is the angle $\beta$ [see McBean (1972) for further details]. For unstable stratifications the distributions for momentum and heat transfer are different, as indicated on the diagram.

are given (after McBean, 1972). For unstable cases the angle of attack can be $30^{\circ}$ or higher. Note that the angle-of-attack response problem (also called the "cosine response" problem) is a different problem from that of tilt of the sensor. The sensor tilt has been extensively discussed in literature (e.g., Kaimal and Haugen, 1969, 1971; Dyer et al., 1970, 1971).

For sensors such as the sonic anemometer, the angleof-attack response is not a problem, but for propeller anemometers or anemoclinometers (Thurtell et al., 1970) the cosine response must be carefully investigated. The data presented in Fig. 3 allow the scientist to compute the magnitude of the error that will arise if his sensor's cosine response is deficient. If the sensors response is only 0.8 of the true magnitude for a $20^{\circ}$ angle of attack, then some of the flux will be missed for unstable cases. By multiplying the normalized fluxangle distribution $\zeta_{w \alpha}(\beta)$ by the cosine response function $C(\beta)$ and integrating from $-180^{\circ}$ to $180^{\circ}$, the error can be determined, i.e.,

$$
\text { percent error }=100\left[1-\int_{-180}^{180} \zeta_{w \alpha}(\beta) C(\beta) d \beta\right] .
$$

This error will also depend on stability.

\section{Gill propeller anemometer}

The Gill propeller anemometer is a relatively inexpensive and easy-to-use instrument for measuring fluctuations in the vertical velocity. The instrument was first reported on by Holmes et al. (1964) and has since been used in several studies of the turbulent fluxes (e.g., Dyer et al., 1967; McBean, 1968; Hicks, 1969). For along-axis winds the instrument is reported to have a distance constant of about $1.1 \mathrm{~m}$ for the 9-inch diameter, 4-blade configuration ${ }^{1}$. For winds not parallel to the propeller axis the distance constant increases with wing angle. ${ }^{2}$ The distance constants become $1.13 \mathrm{~m}$ at $30^{\circ}, 1.3 \mathrm{~m}$ at $40^{\circ}, 1.45 \mathrm{~m}$ at $60^{\circ}, 1.6 \mathrm{~m}$ at $70^{\circ}$ and $\sim 2.2 \mathrm{~m}$ at $80^{\circ}$. The starting speed is $\sim 15 \mathrm{~cm} \mathrm{sec}^{-1}$. The frequency response of this type of sensor is dependent on the wind speed along the axis of the propeller so the response time of the sensor will always be changing, i.e., it will depend on the instantaneous vertical wind. In order to determine the effective response time or frequency cut-off of the sensor, two Gill propeller anemometers were compared with a sonic anemometer. ${ }^{3}$ It has been suggested that the Gill anemometer needs a cylindrical shaft extender on top to give it symmetry in order to improve its cosine response and reduce the stall region. In this experiment, a sonic anemometer (S.A.), a Gill anemometer with a $10-\mathrm{cm}$ shaft extender (G.A.1), and a Gill anemometer without a shaft extender (G.A.2) were mounted side by side along a line perpendicular to the mean wind direction. The lateral separation between each instrument was about $40 \mathrm{~cm}$. In Fig. 4, the vertical velocity spectra for the sonic ane-

\footnotetext{
${ }^{1}$ R. M. Young Có. technical information.

${ }^{2} \mathrm{G}$. C. Gill, private communication.

s Kaijo Kenki, model PAT-311.
} 


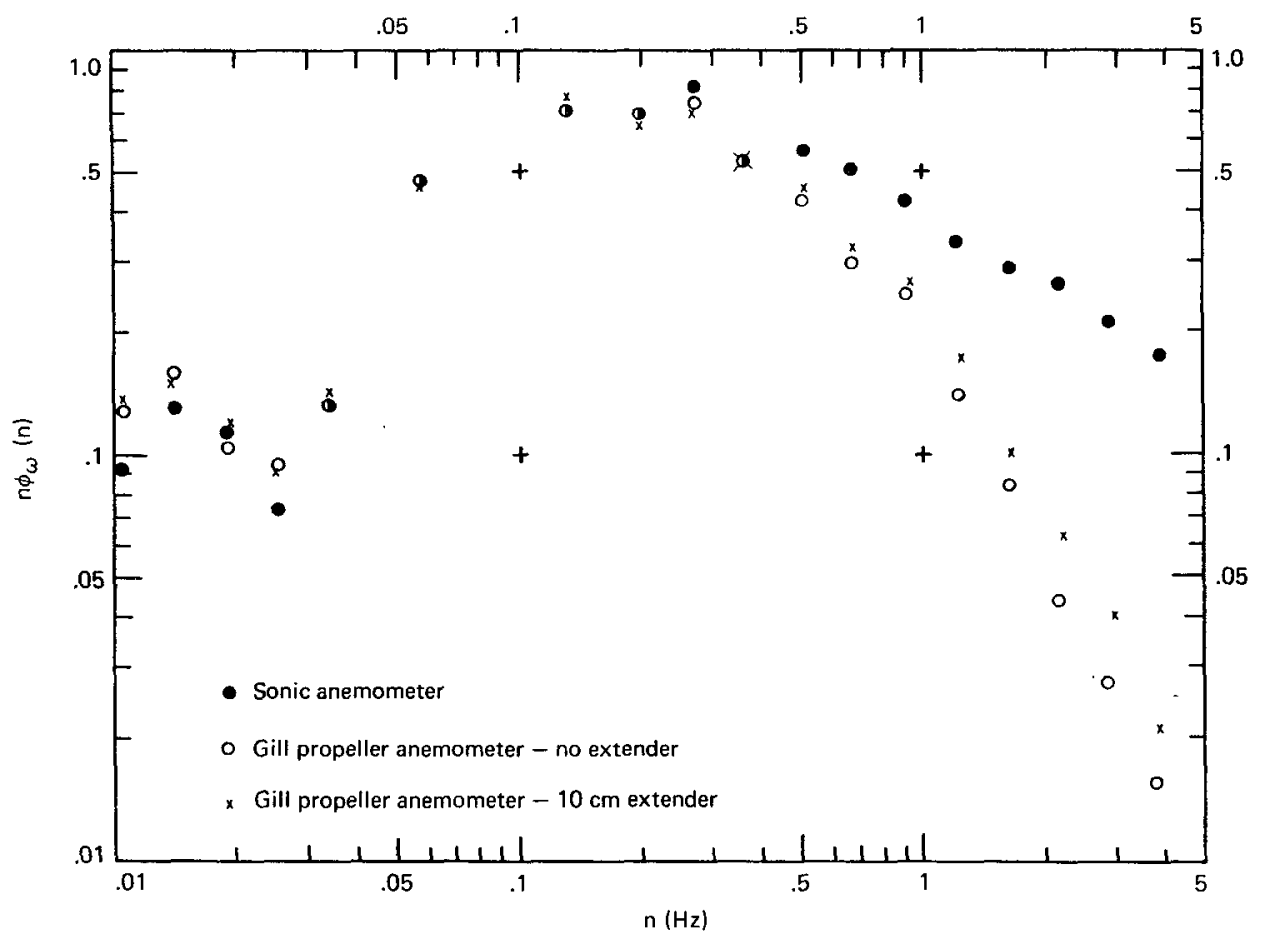

FIg. 4. Comparison of the spectra of the vertical velocity fluctuations as measured with the Kaijo Kenki ultrasonic anemometer and two Gill propeller anemometers, one with a 10-cm shaft extender and one without.

mometer and the two Gill propeller anemometers are compared. For frequencies below $n=0.5 \mathrm{~Hz}$ the spectra agree quite well. At higher frequencies the Gill anemometers are damping out some of the oscillations. The power in the spectra of the Gill anemometers is down by a factor of 2 at $1 \mathrm{~Hz}$ and by a factor of 4 at $2 \mathrm{~Hz}$. Applying the criterion of specifying the frequency response as the frequency at which the power is down by a factor of 2, implies a frequency response for the Gill anemometer of $1 \mathrm{~Hz}$. The mean horizontal wind speed for this case was $6 \mathrm{~m} \mathrm{sec}^{-1}$ while the $1-\mathrm{Hz}$ frequency response corresponds to an effective mean axial wind speed of $1 \mathrm{~m} \mathrm{sec}^{-1}$. An alternate way of considering this is to note that the effective mean elevation angle is about $10^{\circ}$ which corresponds to a wind $80^{\circ}$ off axis. Thus, the distance constant is $\sim 2.2 \mathrm{~m}$ or twice the value for an along axis wind. By applying the dynamic response curve relating amplitude ratio to gust wavelength (supplied by R. M. Young Co.), one can deduce the frequency response for a given wind. The results agree well with the power spectrum comparison. This points out that the effective mean axial wind speed which should be used in computing the frequency response is much less than the mean horizontal wind speed. Because the site for this experiment was far from homogeneous, the intensity of the vertical velocity fluctuations was higher than over homogeneous terrain; hence, the ratio $1 / 6$ for effective wind over mean horizontal wind is probably an over-estimate. Further tests over homo- geneous terrain are needed to determine the response of the sensor more accurately. Note also that there are no significant differences between the spectrum of the Gill anemometer with the extender and that of the Gill anemometer without the extender.

Power spectrum analysis doesn't give all the necessary information on the frequency response. Further information was obtained by computing the coherence and phase relations between the sonic anemometer and each of the Gill anemometers. These results are shown in Fig. 5. For run $a$, the coherence between G.A.1 (with extender) and S.A. doesn't fall off as rapidly as the coherence between G.A. 2 (without extender) and S.A. For run $b$, the extender was switched to G.A. 2 but the S.A.-G.A. 2 coherence was still the same. The conclusion must be that the differences between the coherences S.A.-G.A. 1 and S.A.-G.A. 2 are due to the separation of the sensors and not affected by the extender. Further evidence that the differences are due to space separation is that the G.A. 1-G.A. 2 coherences show similar fall-offs.

The phase relations between the sonic anemometer and the Gill anemometers are also indicated in Fig. 5. These relations are approximately the same for S.A.G.A. 1 and S.A.-G.A. 2 and do not depend on which has the extender. Note that the phase angles increase to about $20^{\circ}$ (with the Gill anemometer lagging) by $0.2 \mathrm{~Hz}$ and to near $60^{\circ}$ by $1 \mathrm{~Hz}$. The Gill anemometers are thus significantly lagging the sonic anemometer 


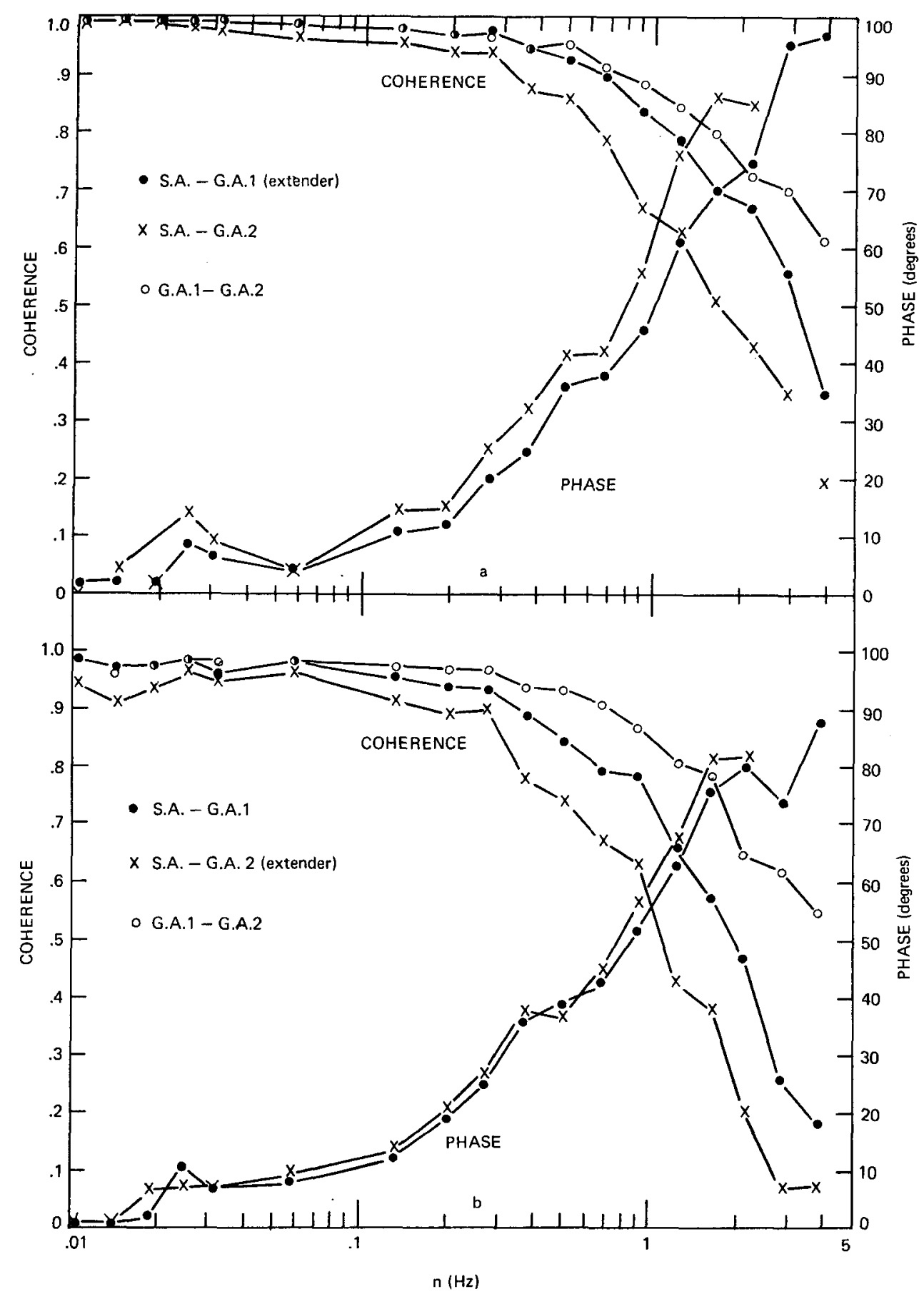

FIG. 5. Coherence and phase relationships between the sonic anemometer (S.A.) and the Gill propeller anemometers (G.A. 1 and G.A. 2). In $a$ the shaft extender was on G.A. 1 (located $40 \mathrm{~cm}$ crosswind from S.A.) and in $b$ the shaft extender was on G.A. 2 (located $40 \mathrm{~cm}$ crosswind from G.A. 1 and $80 \mathrm{~cm}$ from S.A.).

and this will have a marked effect on measured covariances. Corrections could be applied in each case but this can be avoided by matching the phase response of the $\alpha$ sensor to that of the Gill anemometer.

In addition to investigating the frequency response, tests were made to directly determine the anemometer's cosine response. The data published by the manufacturer (R. M. Young Co.) on the sensor showed that its response was $80 \%$ of true cosine response for angles of about $45^{\circ}$. The data presented in Fig. 3 showed that angles of $30^{\circ}$ can be expected for $z / L=-0.5$ and presumably even larger angles would occur for higher in- 


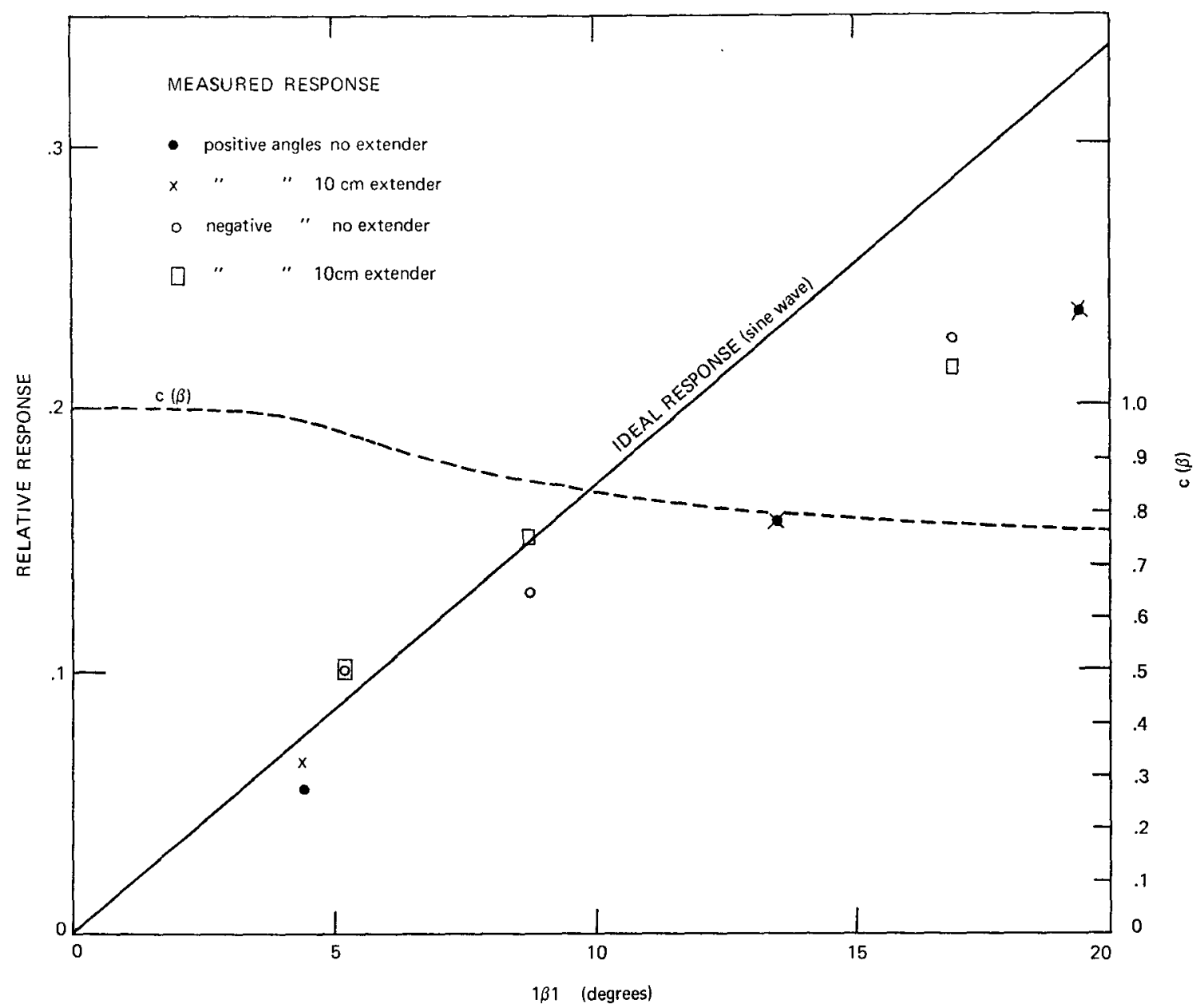

FIG. 6. Data on the angle-of-attack or cosine response of a Gill propeller anemometer, both with and without a 10-cm shaft extender. The solid line is the ideal cosine response and the points indicate the measured response. The dashed curve is the cosine response function, $C(\beta)$, as a function of angle. Note that for the definition used here, the angle between the total wind vector and the horizontal plane, the ideal cosine response function is actually $w / \mathrm{v}=\sin \beta$.

stabilities. A wind-tunnel experiment was conducted to test the anemometer's response to steady winds for different angles of attack, both positive and negative and both with and without the shaft extender. The results (Fig. 6) show that the response deviates significantly from cosine response and indicates again that the cylindrical shaft extender has no significant effect on the anemometer's response. The ratio of actual response to cosine response (Fig. 6) decreased as the angle increased until it became about constant at 0.78 for angles of $\sim 20^{\circ}$. These results agree quite well with a calibration made by G. C. Gill (private communication). The next question is "how important an error is this?." Application of the response ratio of Fig. 6 to the results in Fig. 3 using (4) leads to flux measurement errors of $8 \%$ for both momentum and heat transfer for nearneutral cases and $11 \%$ for heat transfer and $14 \%$ for momentum transfer for $z / L=-0.5$. It was assumed that the response ratio was 0.78 for angles $>20^{\circ}$. In each case, the measured flux would be the given per- centage less than the actual flux. For stable stratifications, the errors due to lack of cosine response are much less but the frequency response errors become very large because of the shift of the cospectrum to higher frequencies which is further worsened by the light winds that usually accompany stable stratifications. The errors due to lack of cosine response must be added to the errors due to frequency response discussed earlier. Thus, the total error will be larger than the errors due to the individual effects.

An example of the error computation can now be given. Consider a system that uses a propeller anemometer with characteristics similar to the ones reported above and which uses electrical RC filters of time constant $100 \mathrm{sec}$ to high-pass filter the data. Since the high-pass filter does not have a sharp cutoff, a representative low-frequency cutoff can be chosen as about $n_{L}=0.0016 \mathrm{~Hz}$, the frequency for $50 \%$ transmission. If the sensor is operated at $4 \mathrm{~m}$ height and the wind speed 
is $6 \mathrm{~m} \mathrm{sec}^{-1}$, then $n_{h}=1 \mathrm{~Hz}, f_{h}=0.67$ and $f_{L}=0.001$. For a near-neutral case the curves on Fig. 2 would imply that $2 \%$ of the flux would be lost due to low-frequency filtering and $14 \%$ would be lost due to sensor damping at high frequencies. This assumes that the temperature or humidity sensor is exactly matched to the anemometer in both phase and amplitude response. This would be very difficult to do because the response of the anemometer is wind-speed dependent. The error due to lack of cosine response of the sensor would be about $8 \%$. The total error would then be of order of $25 \%$, which is quite significant.

It should be pointed out here that the Gill propeller anemometers tested were perhaps not in their best operating condition so that its capabilities may have been underestimated. These results were presented as an example of how to determine the likely errors rather than as a critical examination of this particular sensor.

\section{Concluding remarks}

The preceding sections dealt with the frequency and angle-of-attack or cosine response criteria for eddy flux measurements. It was shown for a particular propeller anemometer that the errors in flux measurements would be significant unless proper corrections were applied. The main source of error was the slow response but the lack of cosine response was also important. As was pointed out careful consideration must be given to matching the phase responses of the sensors. This may be very difficult to achieve when the sensors have very different characteristics of response. In particular, the response of propeller anemometers must be considered in terms of the axial wind speed, not in terms of the mean horizontal wind speed. The results presented here should be used in design of both the equipment and the experiment. For best results eddy flux instruments should be used only if the ratio of height of measurement to wind speed make the loss of flux due to frequency response small.

Acknowledgments. The experimental part of this work was conducted while the author was on educational leave at the Institute of Oceanography, University of British Columbia. The assistance of the faculty, staff and students of that institution are much appreciated. Special thanks are due to Prof. G. C. Gill for providing further information on the propeller anemometer.
Thanks are due to Drs. Fanaki, Martin and Polavarapu for their helpful comments and to Mrs. M. Blace for typing the manuscript.

\section{REFERENCES}

Deacon, E. L., 1959: The measurement of turbulent transfer in the lower atmosphere. Advances in Geophysics, Vol. 6, New York, Pergamon Press, 211-228.

Dyer, A. J., B. B. Hicks and K. M. King, 1967: The fluxatron, a revised approach to the measurement of eddy fluxes in the lower atmosphere. J. A ppl. Meteor., 6, 408-413.

- - - and V. Sitaraman, 1970: Minimizing the levelling error in Reynolds stress measurements by filtering. $J$. $A p p l$. Meteor., 9, 532-534.

- - - - and -1971 : Reply (to Kaimal and Haugen). $J$. Appl. Meteor., 10, 339

Hicks, B. B., 1969: A simple instrument for the measurement of Reynolds stress. I. Appl. Meteor., 8, 825-827.

— 1970: The measurement of atmospheric fluxes near the surface: A generalized approach. $J$. A ppl. Meleor., 9, 386-388.

Holmes, R. M., G. C. Gill and H. W. Carson, 1964: A propellertype vertical anemometer. J. Appl. Meteor., 3, 802-804.

Kaimal, J. C., and D. A. Haugen, 1969: Some errors in the measurement of Reynolds stress. J. Appl. Meleor., 8, 460-462.

, and - 1971: Comments on "Minimizing the levelling error in Reynolds stress measurement by filtering." $J . A p p l$. Meleor., 10, 337-339.

_- , J. C. Wyngaard, Y. Izumi and O. R. Cote, 1972: Spectral characteristics of surface layer turbulence. Quart. J. Roy. Meteor. Soc. (submitted).

Lumley, J. L., and H. A. Panofsky, 1964: The Siructure of Atmospheric Turbulence. New York, Interscience, $239 \mathrm{pp}$.

McBean, G. A., 1968: An investigation of turbulence within the forest. J. Appl. Meleor., 7, 410-416.

- 1972: The turbulent transfer mechanisms: A time domain analysis. Quart. J. Roy. Meteor. Soc. (submitted).

-_-, and M. Miyake, 1972: Turbulent transfer mechanisms in the atmospheric surface layer. Quart J. Roy. Meteor. Soc, 98, 383-398.

Miyake, M., R. W. Stewart and R. W. Burling, 1970: Spectra and cospectra of turbulence over water. Quart. J. Roy. Meteor. Soc., 96, 138-143.

Monin, A. S., and A. M. Obukhov, 1954: Basic laws of turbulent mixing in the ground layer of the atmosphere. Iz. $A \mathrm{kad}$. Nauk. SSSR, Ser. Geofiz., 151, 163-187.

Panofsky, H. A., and E. Mares, 1968: Recent measurements of cospectra for heat-flux and stress. Quart. J. Roy. Meteor. Soc., 94, 581-585.

Pond, S., G. T. Phelps, J. E. Paquin, G. McBean and R. W. Stewart, 1971: Measurements of the turbulent fluxes of momentum, moisture and sensible heat over the ocean. $J$. Atmos. Sci., 28, 901-917.

Thurtell, G. W., C. B. Tanner and M. I. Wesely, 1970: Threedimensional pressure-sphere anemometer system. $J . A p p l$. Meteor., $9,379-385$ 